\title{
The number of smooth 4-manifolds with a fixed complexity
}

\author{
Dave Auckly * \\ June 29, 2018 \\ Department of Mathematics, Kansas State University, Manhattan, KS 66506 USA \\ dav@math.ksu .edu
}

\section{Introduction}

Donaldson demonstrated that topological 4-manifolds could support different smooth structures [?, ?]. After this discovery it was natural to ask how many different smooth structures a given smooth 4-manifold could support. For higher-dimensional manifolds the answer was known - a given (compact) topological manifold of dimension 5 or higher could only support a finite number of different smooth structures. Quickly it was discovered that topological 4-manifolds could admit an infinite number of smooth structures $[?, ?]$. Since then the techniques have improved and we now know many examples of topological 4-manifolds admitting infinitely many different smooth structures [?].

An alternate way to address the question is to ask how the number of smooth structures grows with the complexity of the 4-manifold. Notions of complexity have been used in other settings in low-dimensional topology [?], and a notion of complexity for 4-manifolds was recently introduced in a nice paper of B. Martelli [?]. Martelli proves that the number of homeomorphism classes of simply-connected smooth 4-manifolds with complexity less than $n$ grows as $n^{2}$. This paper addresses the number of diffeomorphism classes of

*This work was partially supported by National Science Foundation grant DMS0604994. 
simply-connected smooth 4-manifolds with complexity less than $n$, proving in particular that this number grows at least as $n \sqrt[3]{n}$. Along the way we construct complete Kirby diagrams for a large family of knot surgery manifolds.

Any smooth 4-manifold admits a handle decomposition. A diagram displaying the attaching regions is called a Kirby diagram; see [?]. Roughly the complexity of a handle decomposition is the sum of the number of disks, strands and crossings in the Kirby diagram. The complexity of a 4-manifold is the minimal complexity of a handle decomposition of the 4-manifold. More precisely, handlebodies are defined recursively with the empty set as the simplest handlebody. A $k$-handle is a copy of $D^{k} \times D^{n-k}$ attached to a handlebody along the so-called attaching region $S^{k-1} \times D^{n-k}$. The result of attaching a handle to a handlebody is a new handlebody. It is not difficult to show that every connected, smooth 4 admits a handle decomposition with exactly one 0-handle. (On the other hand there are topological 4-manifolds that do not admit any handle decomposition.) The attaching regions of the various handles can be put into general position on the boundary of a unique 0-handle. Since this boundary is $S^{3}$ one can assume that the attaching regions of the 1-handles and 2-handles miss one point. Removing this point produces a copy of $\mathbb{R}^{3}$. Each 1 -handle will be attached along a pair of 3-disks. The cores of the 2-handles intersect the boundary of the 0-handle in a compact 1-manifold. (The core of $D^{k} \times D^{n-k}$ is $D^{k} \times\{0\}$.) One can then take a regular projection of this 1-manifold to a plane. The result is a Kirby diagram; see the figures in section 3 for examples. The disks are the components of the attaching regions of the 1-handles. The strands are the components of the intersection of the cores of the 2-handles with the boundary of the 0-handle and the crossings are the crossings in the regular projection.

In order to obtain a lower bound on the growth of the number of smooth structures one must first construct an interesting collection of smooth 4manifolds and compute invariants to show that they are distinct. Next one must construct Kirby diagrams for the manifolds, and finally one will have to do a bit of combinatorics to estimate the number as function of the complexity. This exactly outlines our paper.

Fintushel and Stern gave a way to construct a 4-manifold from a knot, and related the Seiberg-Witten invariants of the 4-manifold to the Alexander polynomial of the knot [?]. Levine constructed a family of knots producing every possible Alexander polynomial [?] completing the first part of the outline. These constructions are reviewed in section 2 below. Akbulut and Auckly independently described handle decompositions of the knot surgery 
manifolds [?,?]. These decompositions are reviewed and simplified in section 3 leaving a bit of combinatorics for section 4 at the end of the paper.

I owe the referee thanks for very helpful comments that pointed out errors in an earlier version of this paper.

\section{The manifolds}

In order to build a family of smooth 4-manifolds one should start with one 4-manifold. We start with an elliptic K3 surface. To be precise let $\mathbb{C} P^{3}:=$ $\left(\mathbb{C}^{4}-\{0\}\right) /(\mathbb{C}-\{0\})$ be complex projective space and define

$$
X:=\left\{\left[z_{0}: z_{1}: z_{2}: z_{3}\right] \in \mathbb{C} P^{3} \mid\left(z_{0}+z_{1}\right)^{4}-z_{1}^{4}+\left(z_{2}+z_{3}\right)^{4}-z_{3}^{4}=0\right\} .
$$

This is the Fermat surface. A simple application of the implicit function theorem proves that it is a smooth 4-manifold. Taken with the projection $\pi: X \rightarrow \mathbb{C} P^{1}$ generically given by $\left[z_{0}: z_{1}: z_{2}: z_{3}\right] \mapsto\left[z_{0}: z_{2}\right]$, this is an elliptic fibration. The inverse image of $T:=\pi^{-1}([2: 1])$ is

$\left\{\left[2 z_{2}: z_{1}: z_{2}: z_{3}\right] \in \mathbb{C} P^{3} \mid 17 z_{2}^{3}+32 z_{2}^{2} z_{1}+24 z_{2} z_{1}^{2}+8 z_{1}^{3}+4 z_{2}^{2} z_{3}+6 z_{2} z_{3}^{2}+4 z_{3}^{3}=0\right\}$.

Further applications of the implicit function theorem demonstrate that this fiber is a smooth, embedded, 2-dimensional torus with tubular neighborhood isomorphic to $T^{2} \times B^{2}$.

Using this torus we can apply the Fintushel-Stern knot surgery construction to obtain a large family of homeomorphic 4-manifolds. Recall how this construction goes. Starting with a knot $K$ in the 3 -sphere one identifies the boundary of $S^{1} \times\left(S^{3}-\operatorname{int}(N(K))\right)$ with $T^{3}$ so that $\mathrm{pt} \times S^{1} \times \mathrm{pt}$ is a meridian of the knot and pt $\times$ pt $\times S^{1}$ is a longitude of the knot in pt $\times S^{3}$. Finally one defines the knot surgery manifold as

$$
X_{K}:=\left(X-T^{2} \times B^{2}\right) \cup_{T^{3}}\left(S^{1} \times\left(S^{3}-\operatorname{int}(N(K))\right) .\right.
$$

The powerful result from [?] is that the Seiberg-Witten invariant of this manifold is given by

$$
\mathrm{SW}_{X_{K}}=\Delta_{K}(t) .
$$

Here $\Delta_{K}$ is the Alexander polynomial of $K$ and $t=\exp (2 \mathrm{PD}[T])$. The main point is that knots with different Alexander polynomials give rise to distinct smooth 4-manifolds.

To go further we need a family of knots with interesting Alexander polynomials. We take the family displayed in figure 1 . 


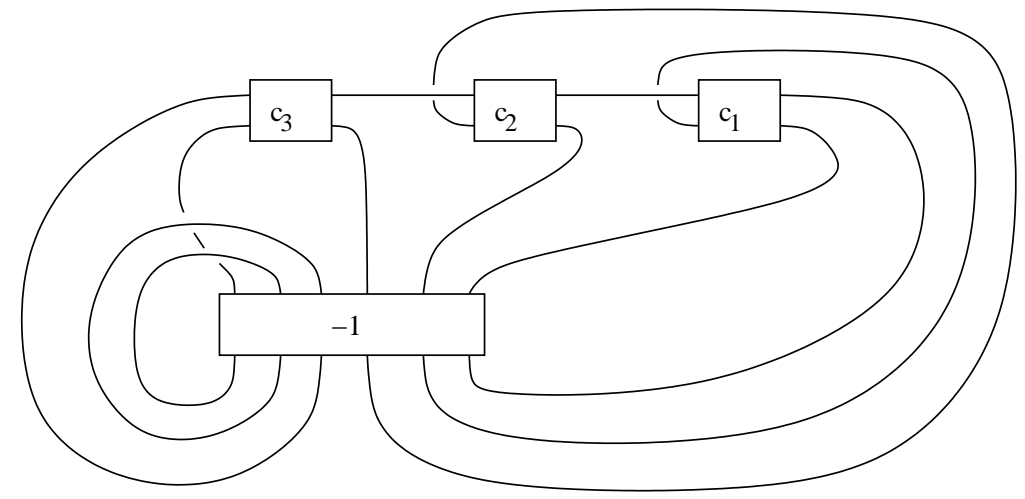

Figure 1: The knot $K\left(c_{1}, c_{2}, c_{3}\right)$

Definition 1. The Levine knots are the knots obtained by generalizing the knot depicted in figure 1 to one with $d$ twist boxes along the top, so that the number of strands passing down through the $(-1)$ twist box is the same as the number that pass up through the twist (-1) twist box. These knots will be denoted by $K\left(c_{1}, c_{2}, \ldots, c_{d}\right)$. We will denote the resulting knot surgery manifold by $X\left(c_{1}, c_{2}, \ldots, c_{d}\right):=X_{K\left(c_{1}, c_{2}, \ldots, c_{d}\right)}$.

This family was originally constructed by J. Levine to characterize all possible Alexander polynomials [?]. Setting $c_{0}:=-1-2 \sum_{k=1}^{d} c_{k}$ one can compute that

$$
\Delta_{K\left(c_{1}, c_{2}, \ldots, c_{d}\right)}(t)=c_{0}+\sum_{k=1}^{d} c_{k}\left(t^{k}+t^{-k}\right) .
$$

If the $(-1)$ twist box in the definition of $K\left(c_{1}, c_{2}, \ldots, c_{d}\right)$ is changed to a $(+1)$ twist box then the value of $c_{0}$ would change to $c_{0}:=1-2 \sum_{k=1}^{d} c_{k}$ and the formula for the Alexander polynomial would remain valid. This produces all possible Alexander polynomials. It will be apparent from the Kirby diagrams that the knot surgery manifolds $X_{-K}$ and $X_{K}$ are diffeomorphic, so for the purposes of this paper there is no loss of generality in using the -1 twist box. An exercise in Rolfsen's book provides the computation of this Alexander polynomial [?]. However, there is a sign error in the exercise so we give a quick sketch of the computation.

First blow up the twist box by adding a $(+1)$-framed surgery component. The resulting link can be isotoped into the projection displayed in figure 2 . In this figure the surgered component (one with twist boxes, red in the electronic 


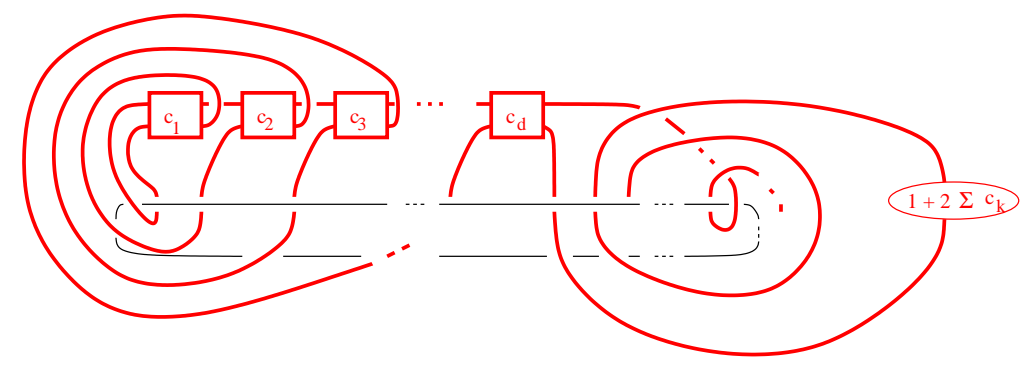

Figure 2: Surgery description of the $K\left(c_{1}, \ldots, c_{d}\right)$ complement

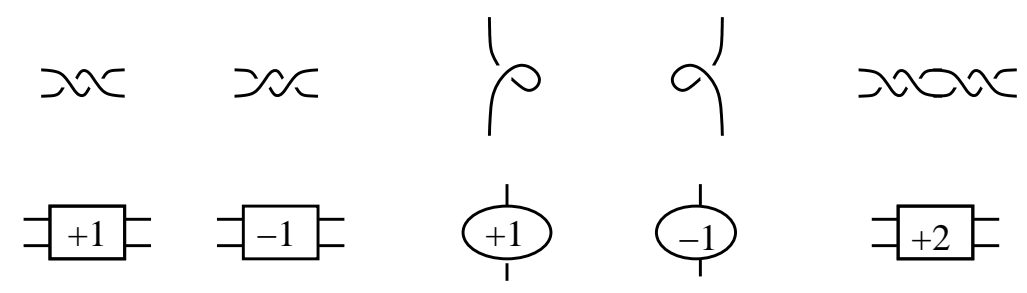

Figure 3: Twist boxes and ovals

version) is drawn using the blackboard framing. To be clear a $(+1)$-twist box, $(-1)$-twist box, (+1)-twist oval, and (-1)-twist oval are displayed in figure 3. Higher order twist boxes and ovals are defined as concatenations.

It is easy to see that the infinite cyclic cover of the knot complement has the surgery description displayed in figure 4. Clearly the first homology of the infinite cyclic cover is generated by the $T^{k} x$ as an abelian group and is generated by $x$ as a $\mathbb{Z}\left[T^{-1}, T\right]$-module. The surgery curves will supply relations. Following the surgery curve labeled with the $x$ from just above the $x$ reading upward one can read off the relation. Since there are $d-1$ crossings before a twist box is encountered, the first twist box will be the $c_{d}$ box meeting the $T^{d} x$ surgery curve. The next box will be the $c_{d-1}$ box on the $T^{d-1} x$ surgery curve, etc.. The last item encountered is the oval that twists the framing. Each positive framing twist contributes a $-x$ to the relation for a total of $c_{0}=-1-2 \sum_{k=1}^{d} c_{k}$. It follows that the first homology of the infinite cyclic cover is a cyclic $\mathbb{Z}\left[T^{-1}, T\right]$-module with relator

$$
\Delta_{K\left(c_{1}, c_{2}, \ldots, c_{d}\right)}(t)=c_{0}+\sum_{k=1}^{d} c_{k}\left(t^{k}+t^{-k}\right) .
$$




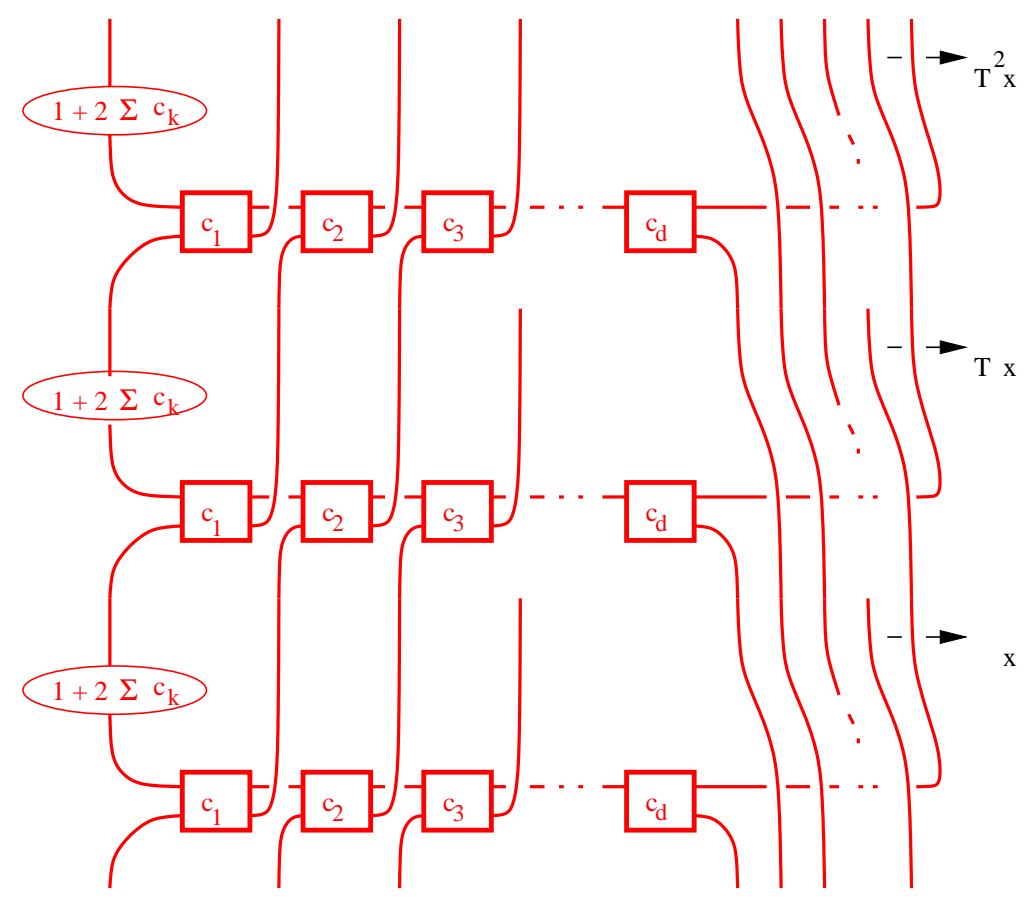

Figure 4: Infinite cyclic cover of the $K\left(c_{1}, \ldots, c_{d}\right)$ complement 


\section{Kirby diagrams}

In this section we construct a simple Kirby diagram for $X\left(c_{1}, c_{2}, \ldots, c_{d}\right)$. This starts with the procedure described in [?, ?]. Since there is a wellknown Kirby diagram for the $K 3$ surface (see [?]) we begin with a Kirby diagram for $S^{1} \times\left(S^{3}-\operatorname{int}\left(N\left(K\left(c_{1}, c_{2}, \ldots, c_{d}\right)\right)\right)\right)$. Clearly this is a union of two copies of $I \times\left(S^{3}-\operatorname{int}\left(N\left(K\left(c_{1}, c_{2}, \ldots, c_{d}\right)\right)\right)\right)$. One obtains a properly embedded 2-disk in $D^{4}$ by taking the interval times the relative pair obtained by removing a small ball containing a standard subarc of any knot $K$. The boundary of the resulting 2-disk is a copy of $K \#-K$. The complement of a tubular neighborhood of this 2-disk is described by putting a dot on the circle representing $K \#-K$. The manifold obtained when this is applied to the unknot is easily seen to be the same as the result of adding a 1-handle to $D^{4}$. This is a generalization of the standard 'dotted circle' notation for 1-handles, and it generalizes in an obvious way to links.

In fact any properly embedded 2-disk in $D^{4}$ in general position with respect to the radius function produces a handle decomposition of the complement of the disk with a 1-handle in the complement for each index 0 critical point of the disk and a 2-handle for each index 1 critical point of the disk etc.; see [?]. The result of this procedure applied to the disk obtained from $K\left(c_{1}, c_{2}, \ldots, c_{d}\right)$ is displayed in figure 5.

To double the handle decomposition here into one for $S^{1} \times\left(S^{3}-\operatorname{int}\left(N\left(K\left(c_{1}, c_{2}, \ldots, c_{d}\right)\right)\right)\right)$ notice that doubling $I \times D^{3}$ amounts to adding a 1-handle to $D^{4}$ and to double the result of adding a 1-handle to $I \times D^{3}$ amounts to adding a 2-handle etc. We call these the doubling handles. This is explained in a bit more detail in [?]. It follows that we could complete the handle decomposition for $X\left(c_{1}, c_{2}, \ldots, c_{d}\right)$ by adding one extra 1-handle, the 2-handles corresponding to the 1-handles in figure 5, the extra 2-handles coming from the decomposition of $K 3$ and the 3 and 4-handles.

We first give the standard Kirby diagram for $K 3$. Applying the procedure that we described in this section to the unknot gives the diagram for $T^{2} \times D^{2}$ on the left of figure 6. The 2-handle in this diagram is taken with the 0 framing. The right side of figure 6 displays the Kirby diagram of the rational elliptic surface. This figure may be obtained by drawing the 1-handle on the right of figure $8.17 \mathrm{a}$ of [?] as a footed handle. The 2 handles that were added to the $T^{2} \times D^{2}$ all come with framing -1 . The 2 -handle that is geometrically unlinked from all of the 1-handles is called the section handle. The other twelve 2-handles are from vanishing cycles. To obtain a Kirby 


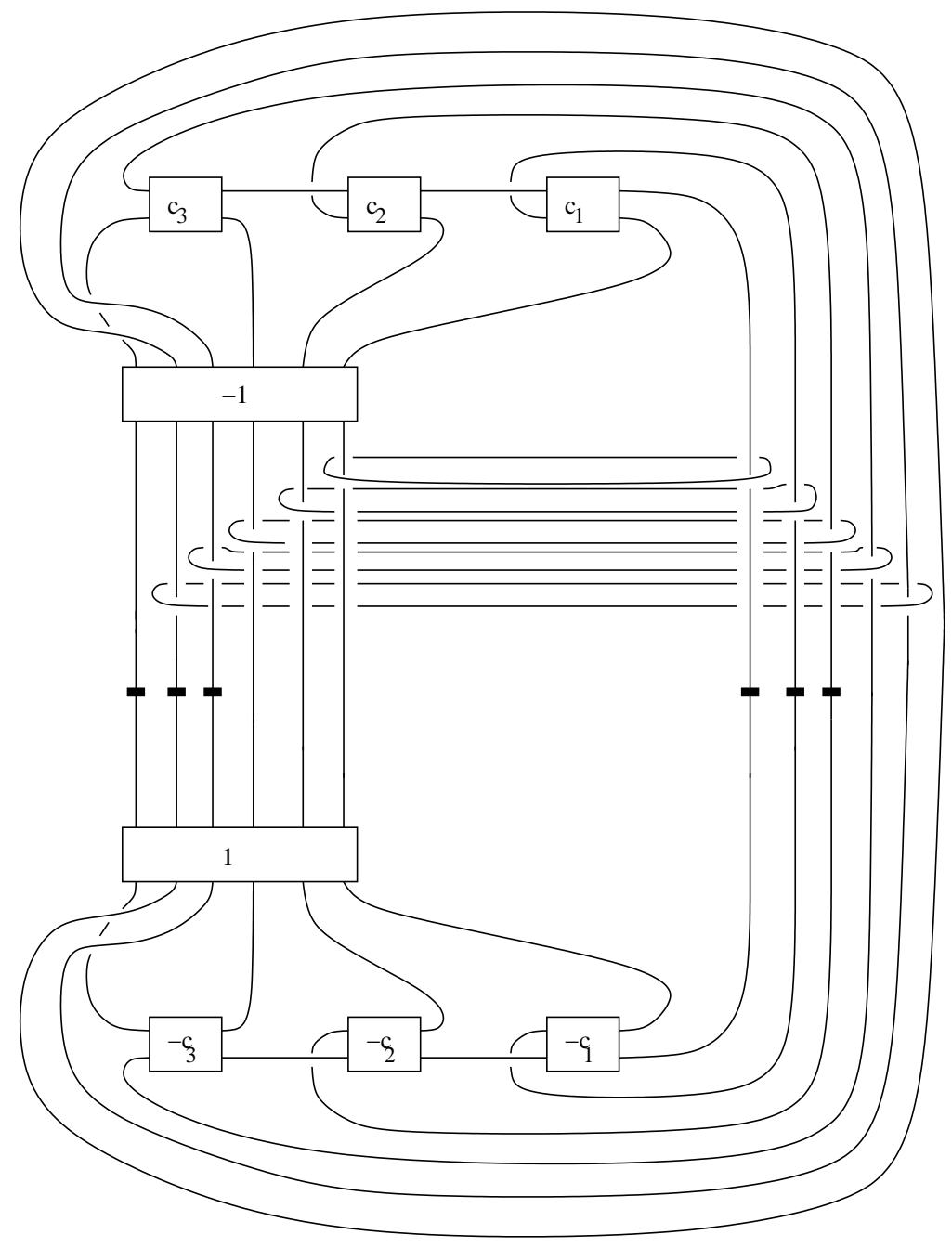

Figure 5: Complement of the $K \#-K$ slice disk 

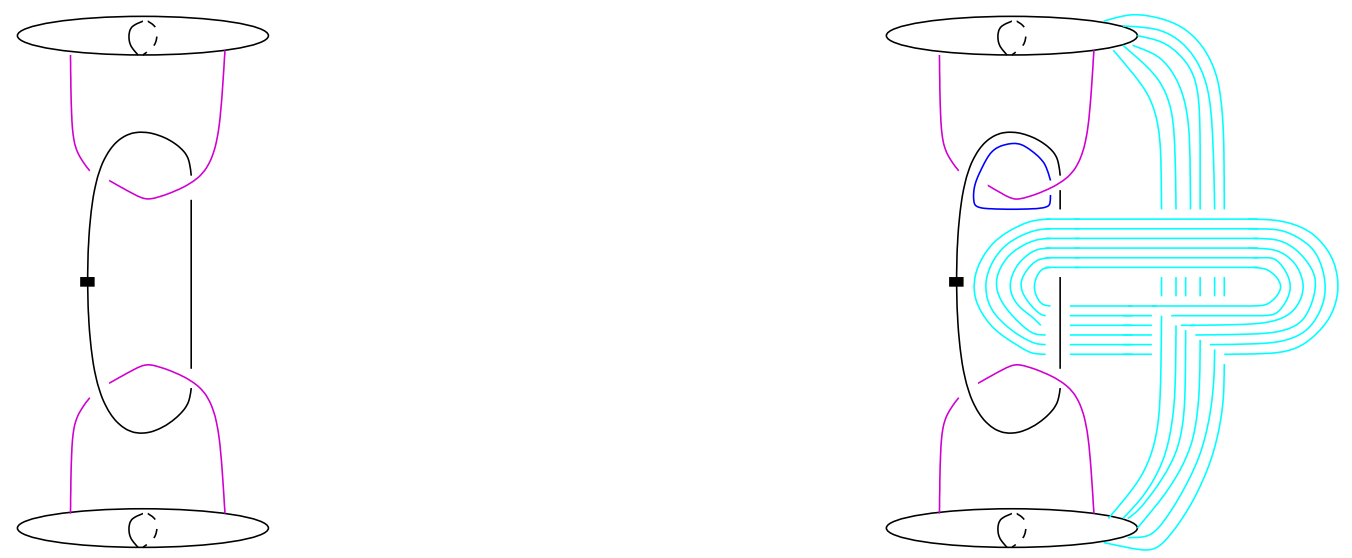

Figure 6: Torus neighborhood and the rational elliptic surface

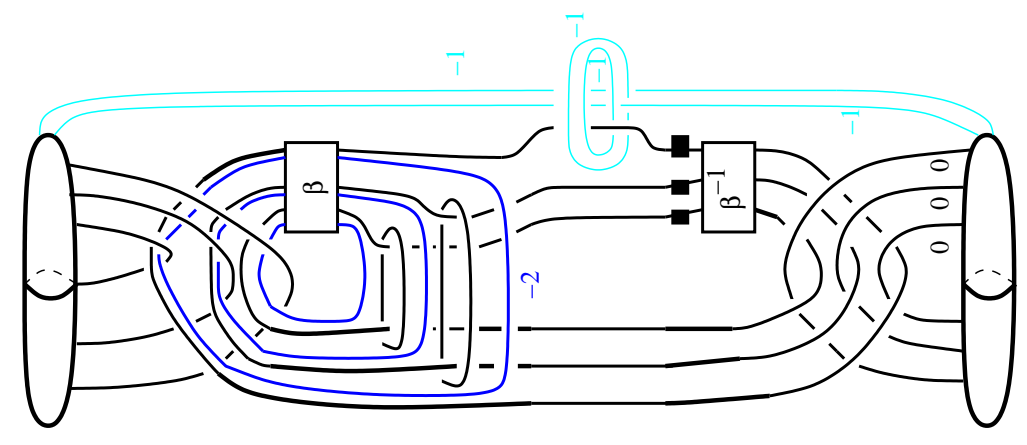

Figure 7: The general knot surgery manifold

diagram for the $K 3$ surface one just changes the framing on the section to -2 and continues to add vanishing cycles in the same pattern until there are a total of 24 vanishing cycles.

To obtain a Kirby diagram for the knot surgery manifolds one just needs to replace the $T^{2} \times D^{2}$ in the $K 3$ diagram with the diagram for $S^{1}$ times the knot exterior. See figure 7 for the general diagram obtained from this procedure. Here we are assuming for simplicity that the knot is the closure of the braid $\beta$. We further assume that the braid is drawn such that the blackboard framing is the zero framing. This can always be done by stabilizing the braid some number of times. Alternately the section handle can be twisted around one of the 1-handles until it represents the longitude of the knot. The braid in the figure only has three strands, but the generalization 

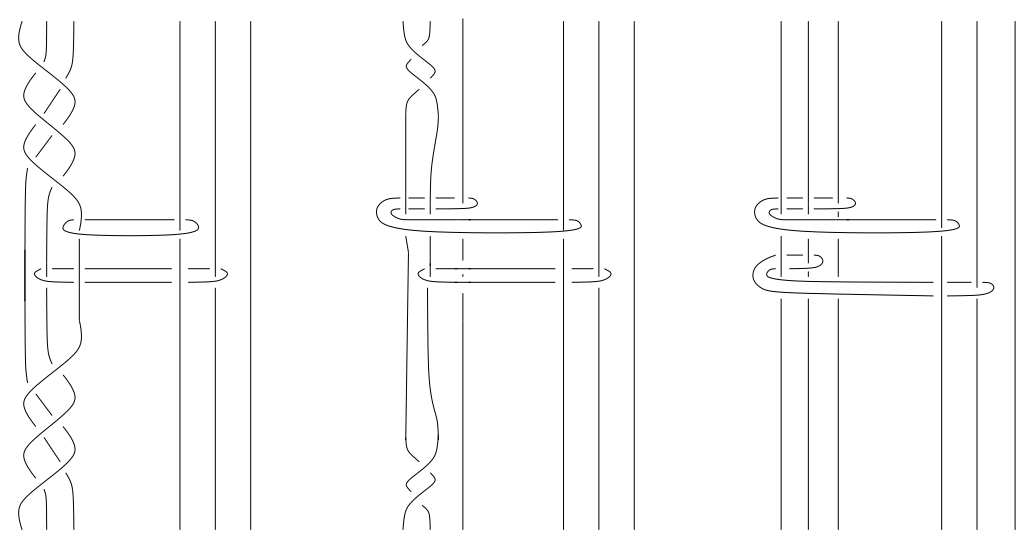

Figure 8: Untwisting

to higher order braids is immediate. In addition this figure only includes four of the 24 vanishing cycles.

The same procedure can be applied to any knot diagram, so we do not have to worry that we don't have a braid presentation for our knot. The result of this procedure applied to the knot $K\left(c_{1}, c_{2}, c_{3}\right)$ will have handles corresponding to doubling the thickened knot complement, section, and vanishing cycles similar to figure 12 but attached to the diagram of the thickened knot complement from figure 5. The problem with this procedure in our case is that we need to represent the 1-handles by the feet of the attaching regions. Thinking about the correspondence between the dotted circle and 1-handle one can see that strands linking the dotted circle in the first representation correspond to strands that pass over the handle in the second representation. It is also clear that the collection of dotted circles representing the 1-handles must form an unlink. This is indeed the case for the diagram in figure 5. However, isotoping the diagram until the dotted circles are in standard position leads to many crossings and a fairly complicated diagram. We will first do a bit of isotopy and then add a few 1/2-handle pairs in order to simplify the diagram further. The result will be figure 12 .

From here forward our goal will be to generate a schematic of a Kirby diagram with no dotted circles and fairly low complexity. A complete Kirby diagram with no dotted circles would be very complicated, but we only need to know enough to estimate the complexity.

The first isotopy will remove the pair of $( \pm 1)$-twist boxes as in figure 8. For the next part of the simplification we will keep track of some of 

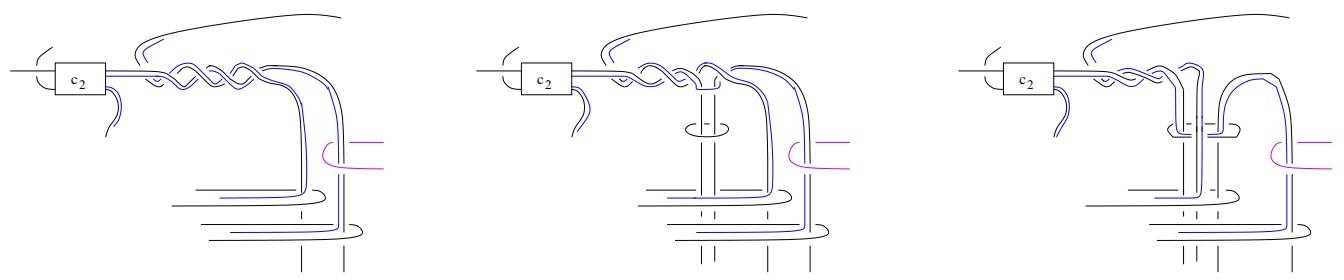

Figure 9: Adding a 1/2 pair

the 2-handles from the $K 3$ side as well. The elliptic fibration $K 3$ has a section. This is a 2-sphere that meets each fiber once. In particular when the $T^{2} \times B^{2}$ is removed a $B^{2}$ is removed from the section, leaving a copy of $D^{2}$ in the complement. Recall that the knot complement circle product is glued so that a longitude of the knot is glued to the boundary of this disk. A neighborhood of this disk becomes a 2-handle attached to the knot complement circle product. This is the (-2)-framed 2-handle in figure 7 . This is why we call this 2-handle the section handle. Figure 9 displays a portion of the handle decomposition from figure 5 after untwisting together with part of the section handle and part of one of the doubling 2-handles, for $c_{1}=2$.

The pair of clasps on the bottom of the figure come from the topmost 2-handle from figure 5 after untwisting. The clasp on the right is part of the doubling 2-handle associated to the 1-handle that it is linking. The loose strands that are mostly parallel to existing 1-handles are parts of the section handle. To complete the figure one can add the reflection of all of the 1handles and the portion of the doubling 2-handle across a horizontal line at the bottom of the figure to the figure. This would produce a larger part of the total Kirby diagram before adding a handle pair. See figure 12 for the result after several handle pair additions and isotopies.

The center diagram can be reflected in the same way without copying the new 2 -handle to obtain the result of adding a $1 / 2$ pair. To see that this is the result of adding a $1 / 2$ pair, slide the more complicated 1-handle passing through the left of the new 2-handle over the 1-handle passing through the right and cancel $1 / 2$ pair. The 2 -handle in the pair is just the meridian of the dotted circle, so all handles that link the dotted circle can be geometrically unlinked via this meridian. Notice that the section handle may slide over the meridian 2-handle, but its position does not change in this process. Also, since the meridian is zero framed and does not link any other 2-handle the 

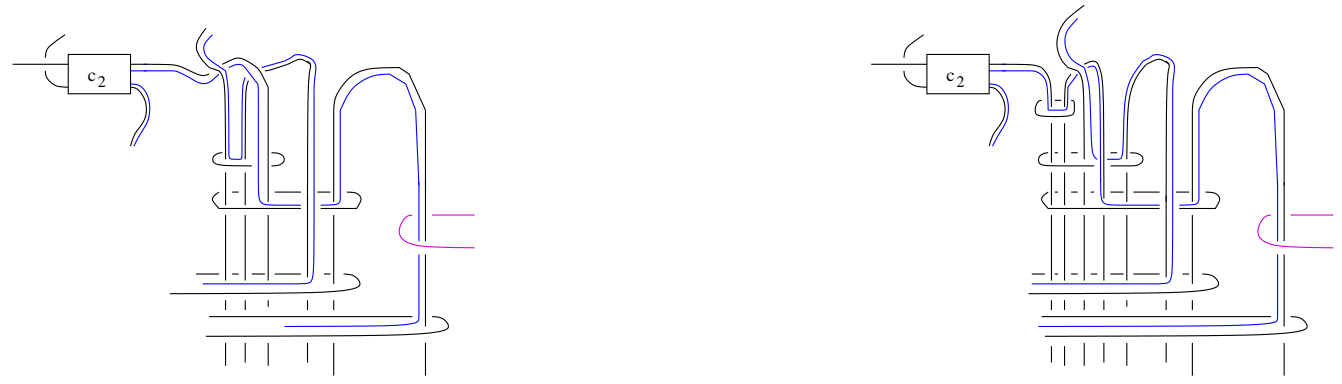

Figure 10: Repeated handle additions

framings on all of the 2-handles are unchanged by this process.

The diagram on the right shows the result after a bit of isotopy. This procedure gets rid of a pair of crossings in the dotted circles at a cost of adding a handle pair and a few more crossings. When we started the section handle entered the crossing of the twist box from the inside. After adding a $1 / 2$ pair and a bit of isotopy the section handle enters the next crossing from the outside. The same procedure can be done when the section handle enters the outside of a crossing. Repeating this procedure, as in figure 10, we can get rid of all of the crossings from the first twist box. In total we add $2 c_{k}-1$ $1 / 2$ pairs to remove a $c_{k}$ twist box when $c_{k}>0$. Notice that for positive $c_{k}$ two crossings can be isotoped away for free, but this does not happen for negative $c_{k}$. For $c_{k}<0$ we can perform a small isotopy (Reidemeister II) to make the twist box look exactly like the figure on the left of figure 9 with all of the crossings in the twist region reversed. This means that we must add $2\left|c_{k}\right|+1$ pairs when $c_{k}<0$. Even though this procedure adds more handles, it is still more efficient than unwinding the 1-handles in the original diagram.

Continuing to add handle pairs produces the diagram in figure 11. (Here we draw the result obtained starting from $c_{k}=3$.) The diagram in the center arises after a bit more isotopy. The diagram on the right arises after a set of handle slides in which the lowest 2-handle is slid over the next highest until all of the new 2-handles take the 'key' shape. The same procedure can be used to remove the remaining twist boxes. The result is displayed in figure 12 for $X(3,3)$, but this figure only includes two of the twenty four vanishing cycles for simplicity. Notice that the section handle forms a copy of the original $K(3,3)$-knot. The twists on the upper left arise because the section handle is attached along a longitude to the original knot that does not link the original knot. Referring to figure 1, we see that each twist box along 

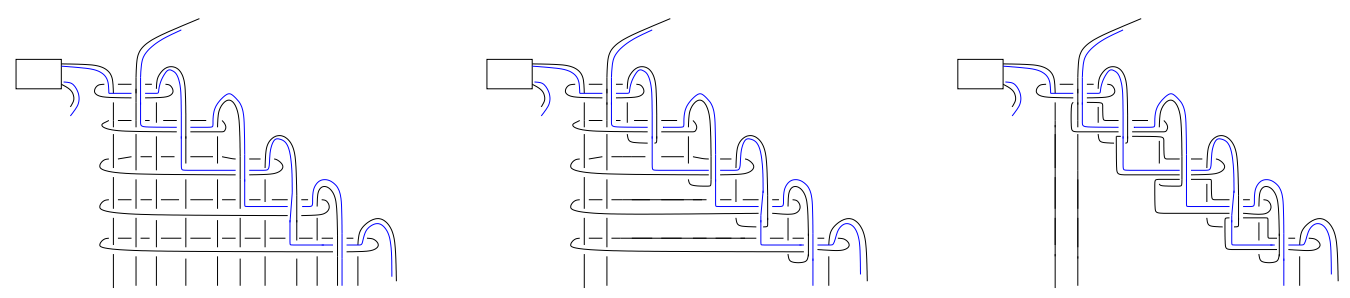

Figure 11: Twist box after handle additions

the top contributes $-2 c_{k}$ to the blackboard framing, and the (-1)-twist box contributes $2 d$ to the blackboard framing. The remaining crossings come in pairs with opposite sign. In order to have the blackboard framing equal to the zero framing, we would have to add an oval representing a writhe of $2 \sum_{k=1}^{d} c_{k}-2 d$. Also notice that we can isotope many of the loops up and slide the new 2-handles over each other as we did in figure 11. In fact the number of loops that hang all the way down from the remains of each twist box is independent of the number of twists in the box.

The only remaining crossings between dotted circles are on the left side of the diagram. These can be removed by a reasonable amount of isotopy. In our diagram of $X(3,3)$ we first stretch the lowest hook further, and then we can untwist. In general we have to stretch the lowest $d-1$ hooks around in the same pattern and then we can isotope. The result is displayed in figure 13. The Kirby diagram for $X\left(c_{1}, c_{2}, \ldots, c_{d}\right)$ is just the natural generalization of the diagram for $X(3,3)$.

\section{The complexity}

The Kirby diagram constructed in the last section is still not suitable for a calculation of the complexity, but it is close. To compute the complexity we need to draw the diagram without dotted circles. We begin this section by describing how to convert a Kirby diagram with knotted circles into one with 1-handles.

The first step is to expand the dual handle decomposition of the slice disks until the collection of dotted circles form an unlink with no crossings. Each dotted circle will separate the plane of the link projection into a bounded component and a non-bounded component. The bounded component will contain a finite number of circles and dotted circles together with a finite 


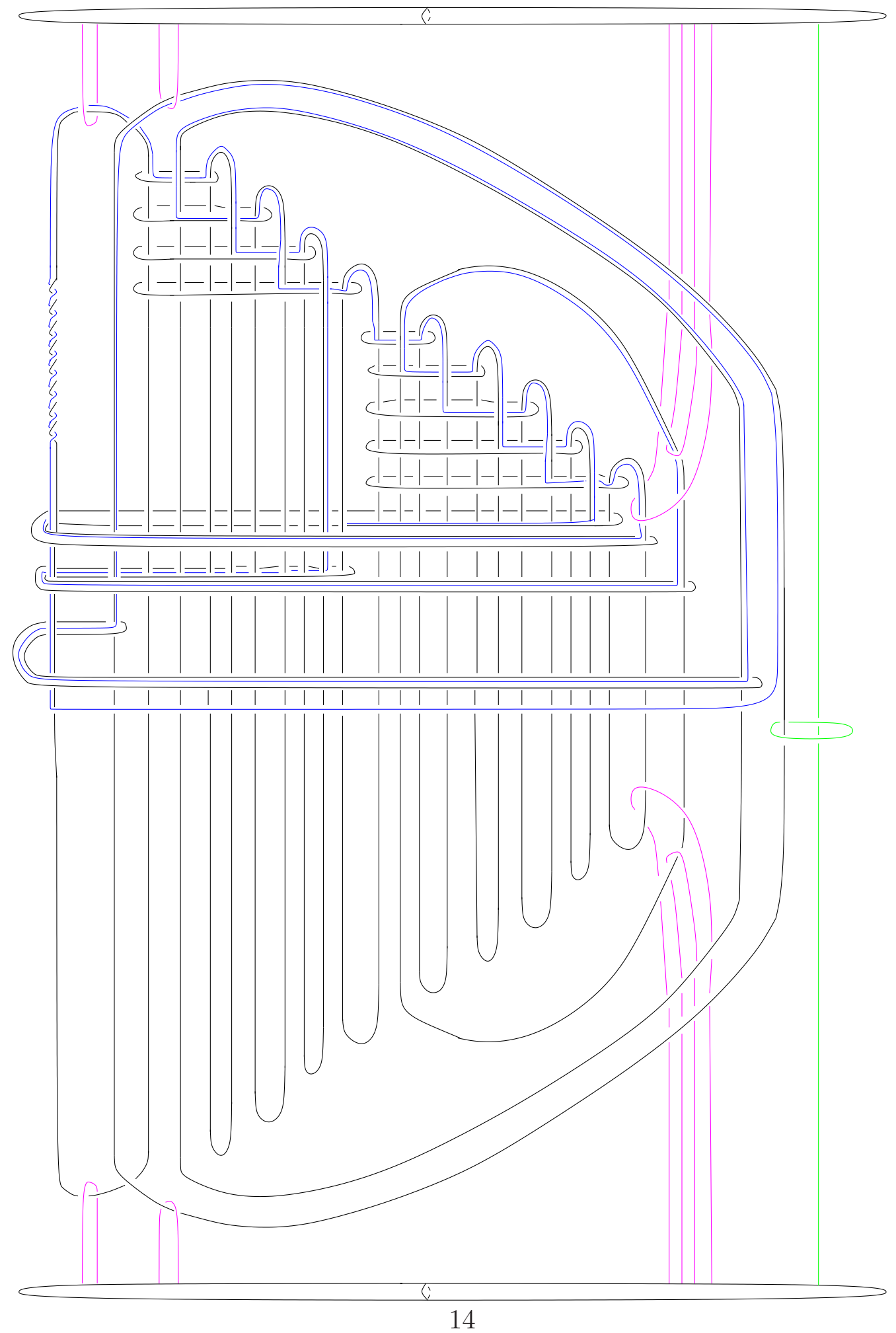

Figure 12: The manifold $X(3,3)$ after some pair additions 


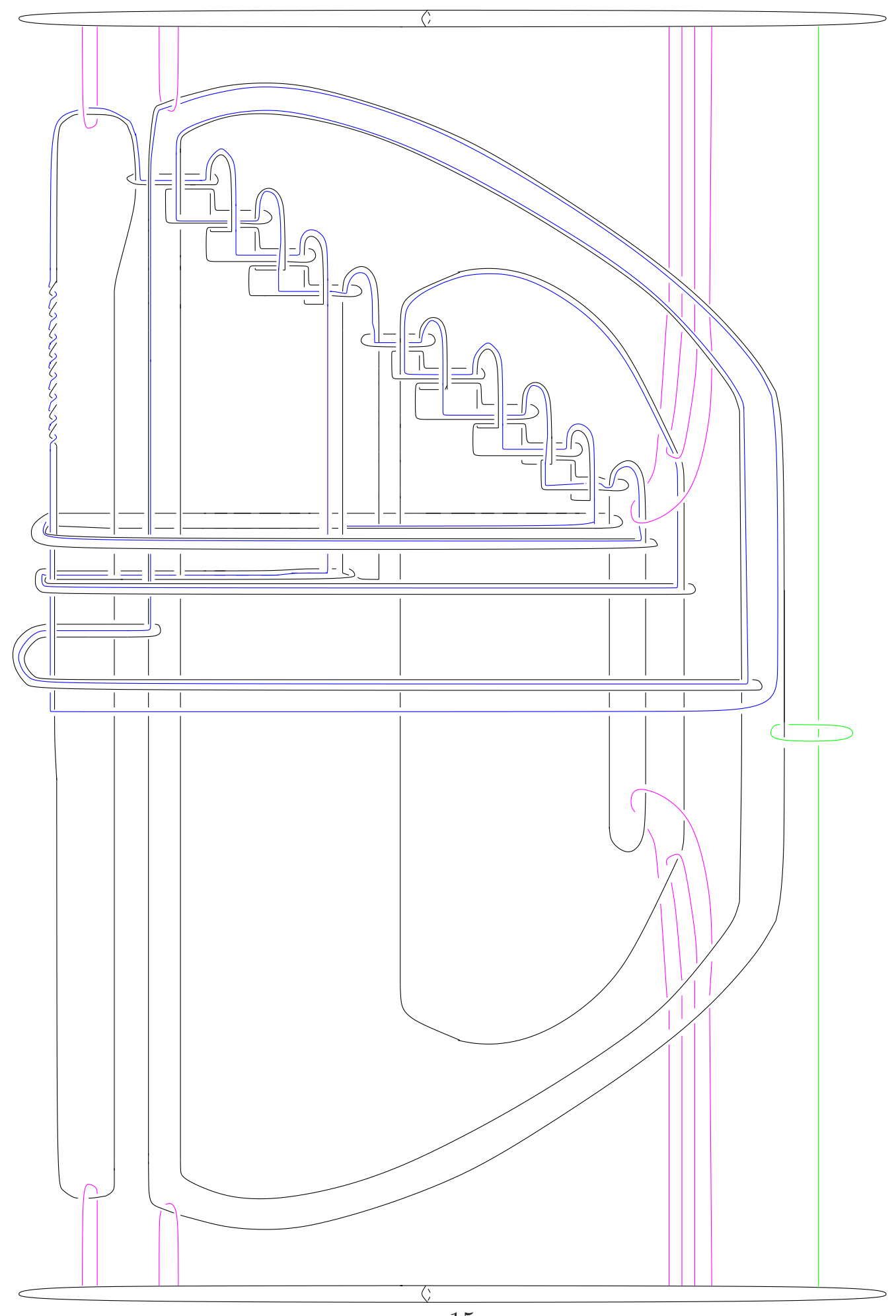

15

Figure 13: Final Kirby diagram for $X(3,3)$ 

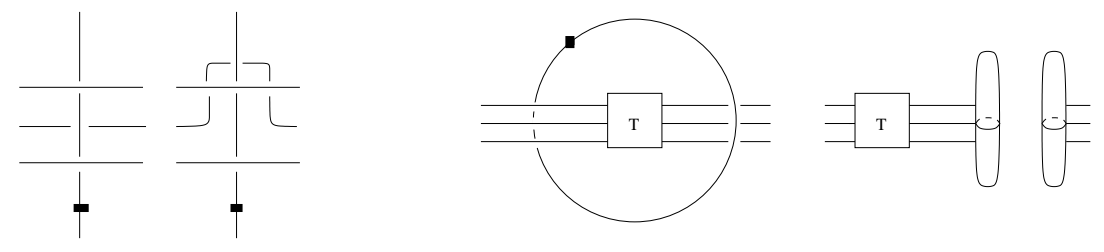

Figure 14: Changing dotted circles into 1-handles

number of arcs interacting in a tangle. We would like all of the over-crossings to be grouped together. Even though this might not be the case, we can arrange for it to be the case by the move depicted on the left of figure 14. Once this is done the dotted circle in standard position can be converted into a 1-handle as on the right of figure 14

What we need at this point is an upper bound on the complexity of $X\left(c_{1}, c_{2}, \ldots, c_{d}\right)$. Recall that the complexity of a manifold is the minimal complexity of a Kirby diagram representing the manifold. We will estimate the complexity of the diagram from figure 13. Recall that the complexity is equal to the number of disks plus the number of strands plus the number of crossings. Clearly the number of disks is equal to twice the number of dotted circles. The initial diagram for the knot complement times an interval had $2 d$ 1-handles. We then added $2\left|c_{k}\right|+1$ 1-handles for each twist box (when $c_{k}$ is positive we could use 2 fewer) and there was one more from doubling. Putting this together shows that

$$
\text { number of disks } \leq 6 d+4 \sum_{k=1}^{d}\left|c_{k}\right|+2 .
$$

Notice that the attaching circle of every 2-handle meets at least one 1handle. To count the number of strands it suffices to count the number of under-crossings between 2-handles and 1-handles. The 2-handles coming from doubling the original 1-handles contribute $O\left(d^{2}\right)$ to this count. Since there is an upper-bound on the number of crossings involving any given meridian 2-handle from the added handle pairs after the last isotopy, each contributes at most a fixed number of strands and the number of these handles is bounded by the total number of twists. Thus the 2-handles in the twist boxes contribute $O\left(\sum_{k=1}^{d}\left|c_{k}\right|+d\right)$ to the count (a total of $d-11$ handles cross the furthest left such handle). Each 'hook' contributes $O(d)$ and there are $O(d)$ hooks for a total of $O\left(d^{2}\right)$. The section handle contributes 
$O\left(\sum_{k=1}^{d}\left|c_{k}\right|+d^{2}\right)$ to the count. This gives

$$
\text { number of strands } \leq O\left(\sum_{k=1}^{d}\left|c_{k}\right|+d^{2}\right) .
$$

When we count the number of crossings we have to take into account the fact that we have to do some moves to group all of the under-crossings of any given dotted circle together, and these moves produce extra crossings in the 2-handles. There is an upper bound on the number of crossings on each 'small' 1-handle in a twist box, and there are $O\left(\sum_{k=1}^{d}\left|c_{k}\right|\right)$ of these 'small' 1-handles. Ignoring the 1-handle on the furthest left for the moment, there are $O(d)$ remaining 1-handles. Each of these crosses $O(d)$ 2-handles, but we might have to push as many as $O(d)$ crossings past $O(d)$ strands as in the left of figure 14, so each of the remaining 1-handles contributes $O\left(d^{2}\right)$ to the crossing count for a total of $O\left(d^{3}\right)$ crossings from these handles. The 1handle on the far left has $O\left(\left|d-\sum_{k=1}^{d} c_{k}\right|\right)$ crossings coming from the writhe correction to the blackboard framing. The over crossings from this area can be grouped together using the move on the left of figure 14 resulting in a total of $O\left(\left(d-\sum_{k=1}^{d} c_{k}\right)^{2}\right)$ crossings. This handle also has $O(d)$ other crossings that can be grouped with the over and under from the writhe area by the same moves contributing $O\left(d^{2}\right)$. Thus

$$
\text { number of crossings } \leq O\left(d^{3}+\sum_{k=1}^{d}\left|c_{k}\right|\right)+O\left(\left(d-\sum_{k=1}^{d} c_{k}\right)^{2}\right) \text {. }
$$

The following estimate follows.

Theorem 1. There are constants $A_{1}, A_{2}$ and $A_{3}$ such that

$$
\text { complexity }\left(X\left(c_{1}, c_{2}, \ldots, c_{d}\right)\right) \leq A_{1} d^{3}+A_{2} \sum_{k=1}^{d}\left|c_{k}\right|+A_{3}\left(d-\sum_{k=1}^{d} c_{k}\right)^{2} .
$$

We can turn this theorem around to obtain a lower bound on the number of smooth classes of simply-connected 4-manifolds with a bounded complexity. First notice that

$$
\#\left\{\left(a_{1}, \ldots, a_{p}\right) \mid a_{k}>0, \sum_{k=1}^{p} a_{k}=m\right\}=\left(\begin{array}{c}
m-1 \\
p-1
\end{array}\right),
$$


as there are $m-1$ 'gaps' in a line of $m$ dots and such a sum corresponds to choosing $p-1$ of the gaps. We can generalize this to estimate the quantity below. By taking the first $[d / 2]$ terms positive and the rest to be negative and taking the sum of the positive terms to be $\frac{1}{2}(m+d)$ we obtain the lower bound

$$
\begin{aligned}
& \#\left\{\left(c_{1}, \ldots, c_{d}\right) \in \mathbb{Z}^{d}\left|\sum_{k=1}^{d}\right| c_{k} \mid \leq m, c_{k} \neq 0, \sum_{k=1}^{d} c_{k}=d\right\} \\
& \geq \#\left\{\left(z_{1}, \ldots, z_{[d / 2]}\right) \mid z_{k}>0, \sum_{k=1}^{[d / 2]} z_{k}=\frac{1}{2}(m+d-2)\right\} \\
& \cdot \#\left\{\left(w_{1}, \ldots, w_{d-[d / 2]}\right) \mid w_{k}>0, \sum_{k=1}^{d-[d / 2]} w_{k}=\frac{1}{2}(m-d-2)\right\} \\
& \geq\left(\begin{array}{c}
\frac{1}{2}(m+d-4) \\
{[d / 2]}
\end{array}\right)\left(\begin{array}{c}
\frac{1}{2}(m-d-4) \\
d-[d / 2]
\end{array}\right) .
\end{aligned}
$$

Here this estimate is valid even if $m$ is not congruent to $d$ mod 2 or even not an integer (this is why we included the extra -2 terms).

Continuing, we see that

$$
\begin{aligned}
& \#\left\{\left(c_{1}, \ldots, c_{d}\right) \mid \text { complexity }\left(X\left(c_{1}, c_{2}, \ldots, c_{d}\right)\right) \leq n\right\} \\
& \quad \geq \#\left\{\left(c_{1}, \ldots, c_{d}\right) \mid \text { complexity }\left(X\left(c_{1}, c_{2}, \ldots, c_{d}\right)\right) \leq n, \sum_{k=1}^{d} c_{k}=d\right\} \\
& \quad \geq \#\left\{\left(c_{1}, \ldots, c_{d}\right)\left|\sum_{k=1}^{d}\right| c_{k} \mid \leq\left(n-A_{1} d^{3}\right) / A_{2}, c_{k} \neq 0, \sum_{k=1}^{d} c_{k}=d\right\} .
\end{aligned}
$$

Now pick $d=2\left[\frac{1}{4}\left(n / A_{1}\right)^{1 / 3}\right]$ and continue the estimate for large $n$

$$
\begin{aligned}
& \geq\left(\begin{array}{c}
A_{2}^{-1} n / 2-4 A_{1} A_{2}^{-1}\left[\frac{1}{4}\left(n / A_{1}\right)^{1 / 3}\right]^{3}+\left[\frac{1}{4}\left(n / A_{1}\right)^{1 / 3}\right]-2 \\
{\left[\frac{1}{4}\left(n / A_{1}\right)^{1 / 3}\right]}
\end{array}\right) \\
& \cdot\left(\begin{array}{c}
A_{2}^{-1} n / 2-4 A_{1} A_{2}^{-1}\left[\frac{1}{4}\left(n / A_{1}\right)^{1 / 3}\right]^{3}-\left[\frac{1}{4}\left(n / A_{1}\right)^{1 / 3}\right]-2 \\
{\left[\frac{1}{4}\left(n / A_{1}\right)^{1 / 3}\right]}
\end{array}\right) \\
& \geq\left(\begin{array}{c}
A_{2}^{-1} n / 3 \\
A_{1}^{-1 / 3} n^{1 / 3} / 5
\end{array}\right)^{2} \geq n^{c \sqrt[3]{n}} .
\end{aligned}
$$


To obtain the last estimate we use $\left(\begin{array}{l}n \\ k\end{array}\right) \geq(n / k)^{k}$. We summarize this in the following theorem.

Theorem 2. There is a constant $c$ so that for large $n$ the number of diffeomorphism classes of simply-connected manifolds homeomorphic to K3 having complexity less than or equal to $n$ is at least $n^{c \sqrt[3]{n}}$.

This should be compared with the following result of Martelli.

Theorem 3 (Martelli). The number of homeomorphism classes of simplyconnected 4-manifolds having complexity less than or equal to $n$ is between $(1 / 4) n^{2}$ and $(5 / 16) n^{2}$.

It should come as no surprise that the number of diffeomorphisms grows much faster (faster than any polynomial) than the number of homeomorphism classes (quadratic).

A more careful analysis of the crossings showing that the number of crossings is $O\left(d^{2}+\sum_{k=1}^{d}\left|c_{k}\right|\right)$ might be possible. This estimate would show that the number of diffeomorphism types grows at least as $n^{c \sqrt{n}}$. It is harder to imagine improving the bound much more than that with the same techniques. Other constructions lead to similar combinatorics: using other elliptic surfaces just changes the number of vanishing cycles by a constant; distinguishing the result of several knot surgeries on distinct fibers is easiest if each occurs in a $c$-neighborhood in which case the complexity would appear to grow quadratically in the number of knot surgeries; link surgeries also appear to have similar combinatorics.

Martelli showed that the number of diffeomorphism classes of smooth 4manifolds having complexity no greater than $n$ grows no faster than $n^{C n}$ for some constant $C$. It may be that this is the right growth rate for simplyconnected diffeomorphism types. To prove this one would need to find a considerable simplification of the Kirby diagrams presented here or find a different family of manifolds with simple Kirby diagrams. Alternately one could look for new 4-manifold invariants. There are many more knots than the ones that we have considered; however, the ones that we considered take every possible value of the Alexander polynomial, so it is impossible to get a Seiberg-Witten invariant other than the ones we have here with a single knot surgery on a fiber of a $K 3$.

It is interesting to ask the similar questions for 4-manifolds with additional structure. For example to address symplectic 4-manifolds it is natural 
to consider knot surgery with fibered knots. This is similar in spirt to the work of Baldridge and Kirk addressing how large a symplectic 4-manifold with given fundamental group must be [?].

While it is clear that it is possible to answer a number of questions about the complexity of 4-manifolds, much less is known about 4-manifolds in general than is known about 3-manifolds. Thus questions of complexity are still more relevant in 3-dimensions where more subtle questions can be addressed [?]. 

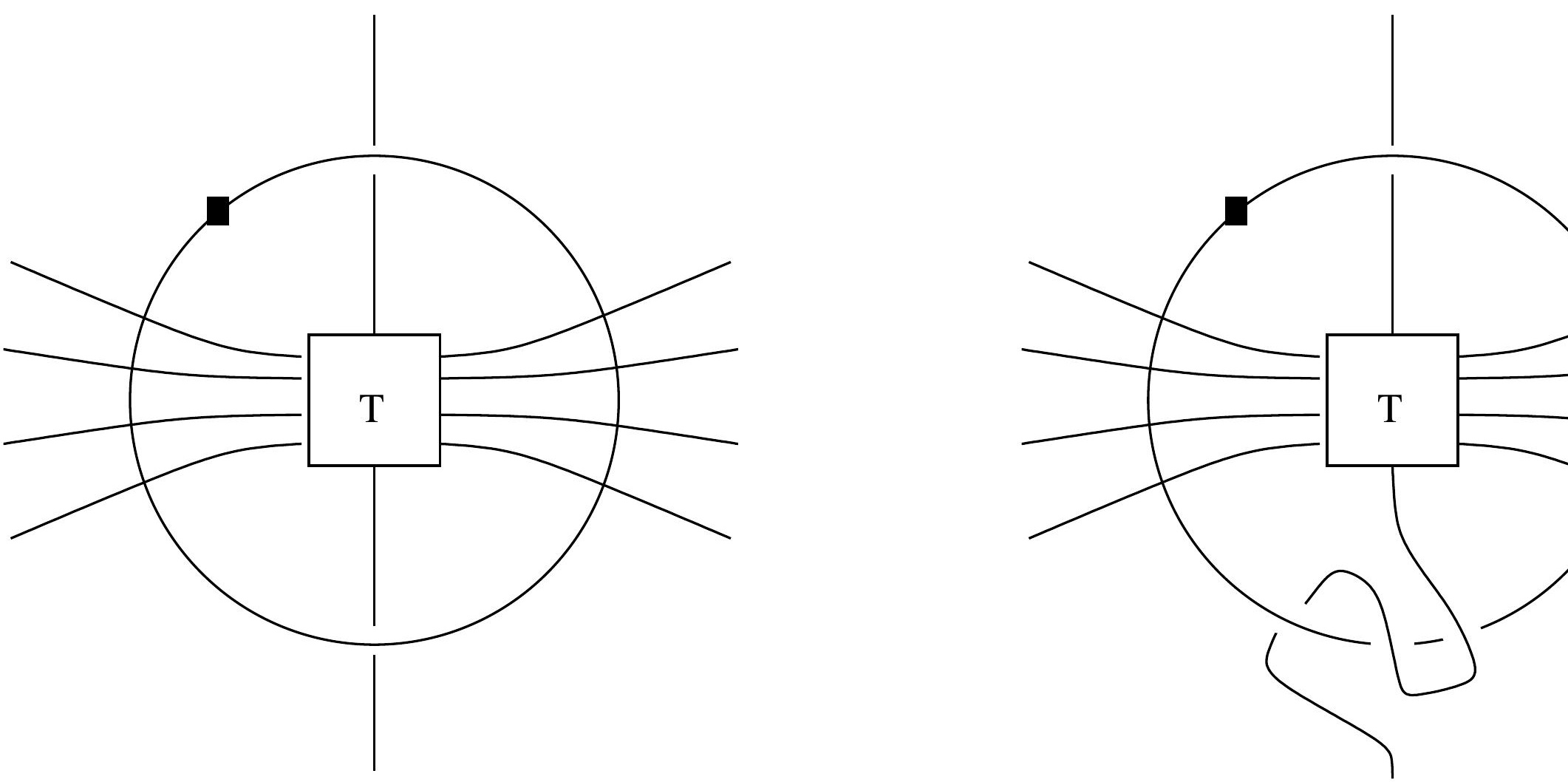

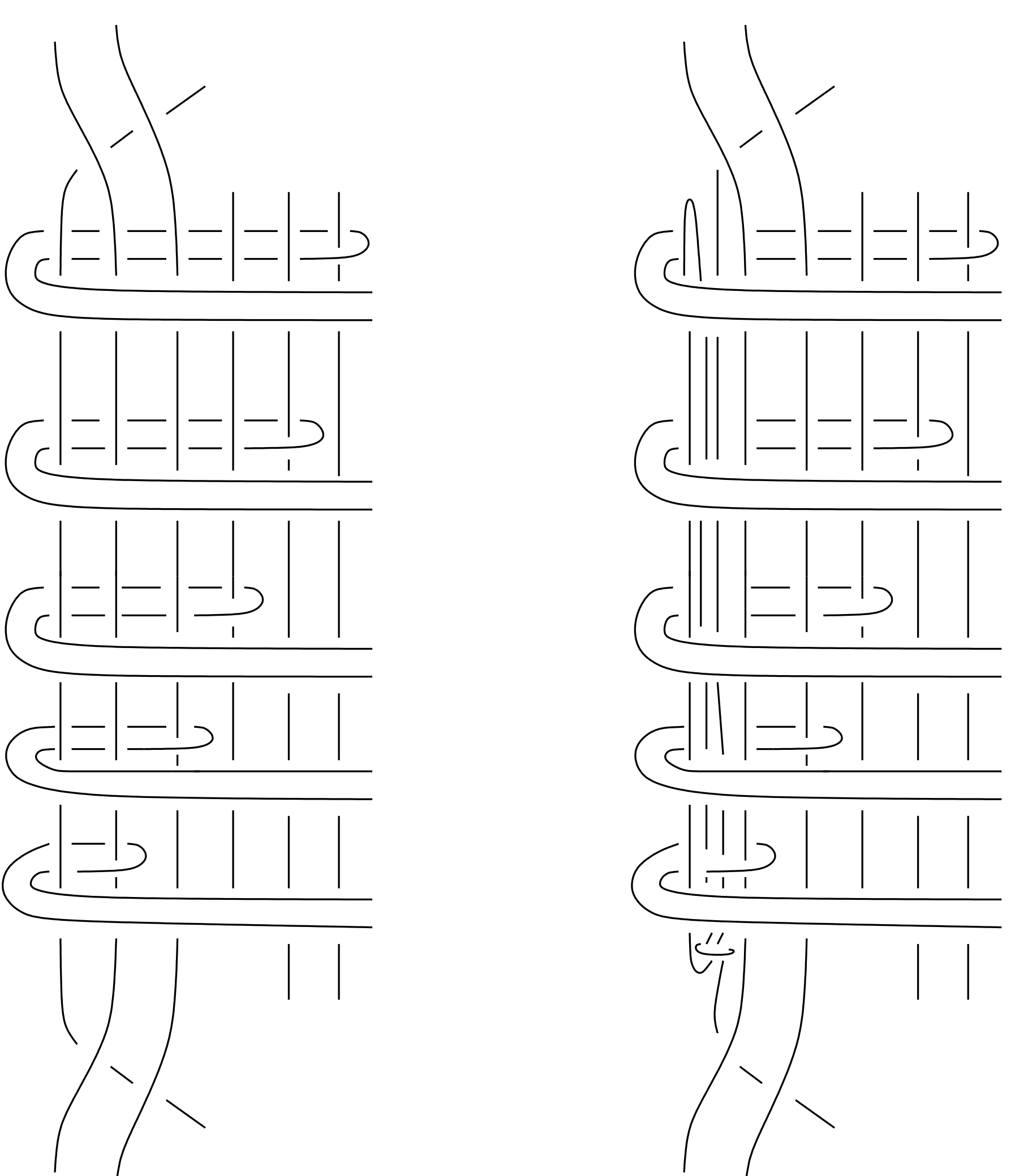


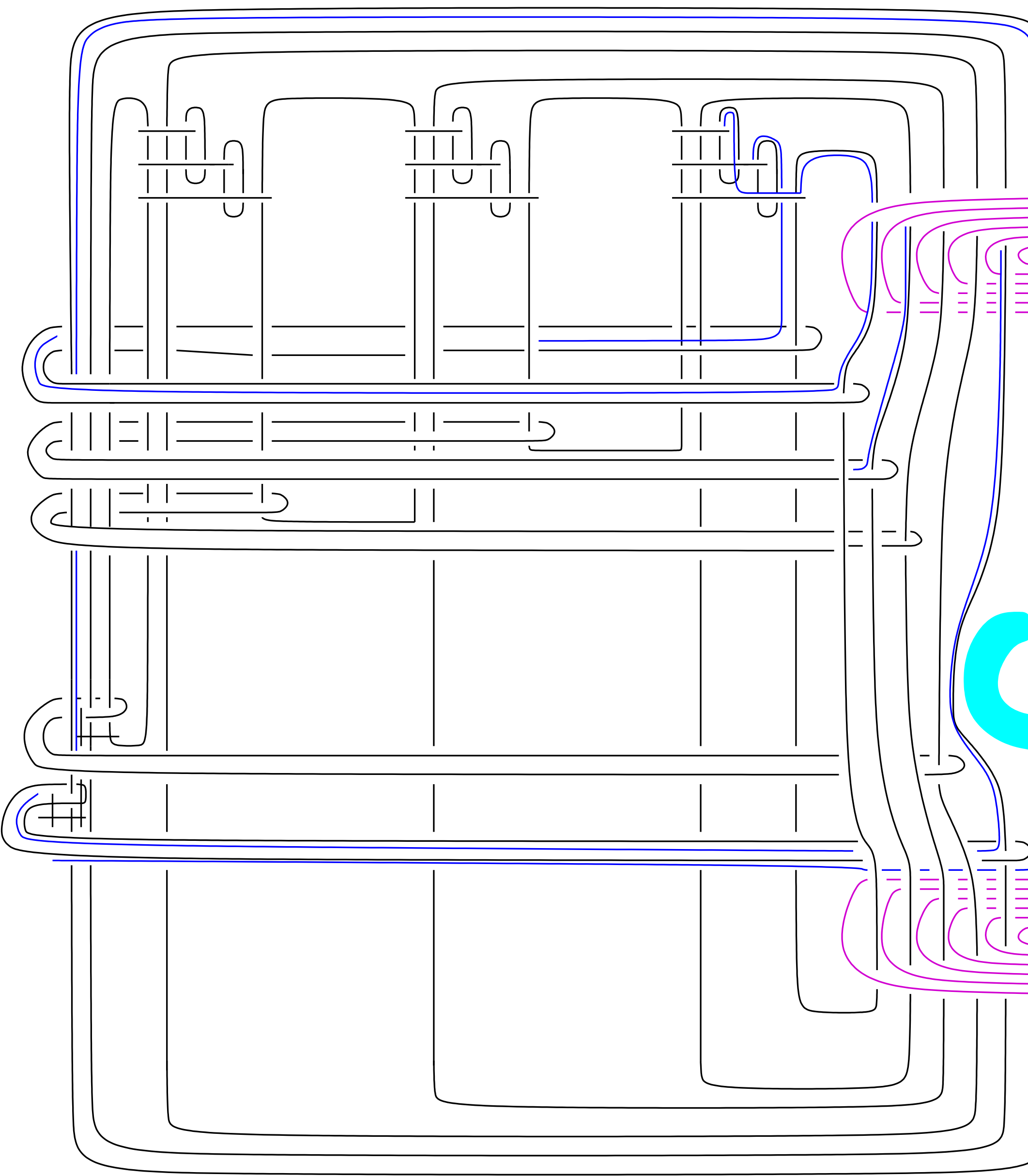

